\title{
Clonogenic long-term survival assay of HCT 116 colorectal cancer cells after treatment with the synthesized diphenyl imidazoline derivatives
}

\author{
Youngshim Lee ${ }^{1} \cdot$ Dongsoo Koh $^{2} \cdot$ Seunghyun Ahn ${ }^{1}$. \\ Young Han Lee ${ }^{3}$ Soon Young $\mathrm{Shin}^{3}$ - Yoongho Lim ${ }^{1}$
}

Received: 8 February 2018/Accepted: 21 February 2018/Published online: 23 March 2018

(C) The Korean Society for Applied Biological Chemistry 2018

\begin{abstract}
Fourteen diphenyl imidazoline derivatives were designed, synthesized, and identified using NMR spectroscopy and high-resolution mass spectrometry. Their cytotoxicities in HCT 116 colorectal cancer cell lines were measured using a clonogenic long-term survival assay and the half-maximal cell growth inhibitory concentration $\left(\mathrm{GI}_{50}\right)$ values were in the range $3.1-58.4 \mu \mathrm{M}$. As the anticancer effects of diphenyl imidazolines were reported to be caused by the inhibition of mouse double minute 2 homolog (MDM2), the inhibitory effects of the most potent derivative on MDM2 were assessed through Western blotting analysis. In silico docking experiments revealed the binding mode between this derivative and MDM2.
\end{abstract}

Keywords Diphenyl imidazolines · Colorectal cancer . MDM2 · Clonogenicity

Youngshim Lee and Dongsoo Koh contributed equally to this work,

Soon Young Shin

shinsy@konkuk.ac.kr

$\triangle$ Yoongho Lim

yoongho@konkuk.ac.kr; yoongholim@gmail.com

1 Division of Bioscience and Biotechnology, BMIC, Konkuk University, Seoul 05029, Republic of Korea

2 Department of Applied Chemistry, Dongduk Women's University, Seoul 02748, Republic of Korea

3 Department of Biological Sciences, Konkuk University, Seoul 05029, Republic of Korea

\section{Introduction}

During the last two decades, various diphenyl imidazolines have been synthesized and their biological activities have been evaluated. Antirheumatic, antidepressant, antiplasmodial, antitrypanosomal, and antimicrobial effects have been reported [1-6]. In addition, their potential as cardiovascular drugs, alpha 1-adrenoceptor antagonists, histone deacetylase-3 inhibitors, and spinal muscarinic M1 receptor activators have been tested [7-10] and their antitumor activity against HL60 cancer cells has also been reported [11].

Colorectal cancer refers to the cancer of the large intestine, colon, and the last part of the colon, the rectum. It is the third most common cancer in the USA, with more than 100,000 patients diagnosed in 2016, and it is the thirdleading cause of cancer-related deaths [12]. The possible treatments include surgery, radiation therapy, and chemotherapy, but research into new chemotherapeutic agents is in progress. There are many methods to measure the anticancer effects of small compounds. One method is the measurement of their cytotoxicity in cancer cell lines [13-15]. Although the clonogenic long-term survival assay requires a time period of several days to obtain results for cytotoxic activities, it can distinguish the cytotoxicities between compounds containing a common skeleton [16]. To find diphenyl imidazolines that show cytotoxicity against colorectal cancer cell lines, 14 diphenyl-imidazoline derivatives were designed and synthesized. Their cytotoxicities in the HCT 116 colorectal cancer cell line were measured using a clonogenic long-term survival assay and their half-maximal cell growth inhibitory concentration $\left(\mathrm{GI}_{50}\right)$ values were observed at the micromolar level. Recently, it was reported that the anticancer effects of 
diphenyl imidazolines were attributable to their inhibitory effects against mouse double minute 2 homolog (MDM2) [17-20]. Therefore, the most potent compound, (4R,5R)-2(naphthalen-2-yl)-4,5-diphenyl-imidazoline, synthesized in this study was assessed for its inhibitory effect on MDM2 by Western blotting analysis. The binding mode between this compound and MDM2 was elucidated using in silico docking. The goal of this research was to study the structure-activity relationships (SARs) to suggest the structural features that contribute to the compound's strong cytotoxicity.

\section{Materials and methods}

\section{Preparation of diphenyl imidazoline derivatives}

(1R, 2R)-(+)-1,2-diphenylethylenediamine (DPEN, $11 \mathrm{mmol}, 2.33 \mathrm{~g}$ ) and $p$-methoxy benzaldehyde $(10 \mathrm{mmol}$, $1.36 \mathrm{~g}$ ) were dissolved in $100 \mathrm{~mL} t-\mathrm{BuOH}$. The mixture was stirred at room temperature for $30 \mathrm{~min}$ after which potassium carbonate $(30 \mathrm{mmol}, 4.14 \mathrm{~g})$ and iodine $(12.5 \mathrm{mmol}, 3.15 \mathrm{~g})$ were added to the mixture. The reaction mixture was stirred at $70{ }^{\circ} \mathrm{C}$ for $3 \mathrm{~h}$. When the completion of the reaction was confirmed by thin layer chromatography, the reaction mixture was quenched with saturated $\mathrm{Na}_{2} \mathrm{~S}_{2} \mathrm{O}_{5}$ solution until the iodine color disappeared. The precipitate was filtered under vacuum and dried. The crude solid was recrystallized from ethanol. Yield 60\%, m.p. 216-218 (white solid). The synthetic process is summarized in Scheme 1. Of 14 derivatives synthesized here, three derivatives $\mathbf{1}, \mathbf{4}$, and 9 have been reported previously.

(4R,5R)-2-(4-methoxyphenyl)-4,5-diphenyl-imidazoline (1) [21] Ivory (18\% yield); $\mathrm{Mp}=216-218{ }^{\circ} \mathrm{C} ;{ }^{1} \mathrm{H}$ NMR $\left(400 \mathrm{MHz}, \mathrm{DMSO}-d_{6}\right) \delta 7.95\left(\mathrm{~d}, J=8.6 \mathrm{~Hz}, 1 \mathrm{H}, \mathrm{H}-2^{\prime}\right)$, 7.43 (m, 2H, H-2/H-6), 7.36 (m, 2H, H-3/H-5), 7.24 (m, $1 \mathrm{H}, \mathrm{H}-4), 7.03\left(\mathrm{~d}, J=8.6 \mathrm{~Hz}, 1 \mathrm{H}, \mathrm{H}-3^{\prime}\right), 4.84$ (d, $J=8.3 \mathrm{~Hz}, 1 \mathrm{H}, \mathrm{Im}-\mathrm{H}-4), 4.63$ (d, $J=8.3 \mathrm{~Hz}, 1 \mathrm{H}, \mathrm{Im}-\mathrm{H}-$ 5), $3.82\left(\mathrm{~s}, 3 \mathrm{H}, 4^{\prime}-\mathrm{OCH}_{3}\right) ;{ }^{13} \mathrm{C}$ NMR $(100 \mathrm{MHz}$, DMSO$\left.d_{6}\right) \delta 162.1$ (Im-C-2), $161.2\left(\mathrm{C}-4^{\prime}\right), 144.2$ (C-1), 128.9 (C2'/C-6'), 128.3 (C-3/C-5), 126.6 (C-4), 126.4 (C-2/C-6),
$122.8\left(\mathrm{C}-1^{\prime}\right), 113.6\left(\mathrm{C}-3^{\prime} / \mathrm{C}-5^{\prime}\right), 79.3$ (Im-C-4), 69.0 (Im-C5), $55.2\left(4^{\prime}-\mathrm{OCH}_{3}\right) ; \quad v_{\max }$ (ATR) 315630242964 $1614 \mathrm{~cm}^{-1}$; HRMS (m/z): Calcd. for $\mathrm{C}_{22} \mathrm{H}_{20} \mathrm{~N}_{2} \mathrm{O}$ $[\mathrm{M}+\mathrm{H}]^{+}:$328.1627, Found. 328.1576.

(4R,5R)-2-(2,4,6-trimethoxyphenyl)-4,5-diphenyl-imidazoline (2) White (43\% yield); $\mathrm{Mp}=124-125{ }^{\circ} \mathrm{C} ;{ }^{1} \mathrm{H}$ NMR $\left(400 \mathrm{MHz}, \mathrm{CHCl}_{3}-d\right) \delta 7.43(\mathrm{~d}, J=8.2 \mathrm{~Hz}, 2 \mathrm{H}, \mathrm{H}-2 / \mathrm{H}-6)$, 7.34 (t, $J=7.1 \mathrm{~Hz}, 2 \mathrm{H}, \mathrm{H}-3 / \mathrm{H}-5), 7.26(\mathrm{~m}, 1 \mathrm{H}, \mathrm{H}-4), 6.16$ (s, 1H, H-3'), 4.84 (s, 2H, Im-H-4/Im-H-5), 3.86 (s, 6H, 2'$\left.\mathrm{OCH}_{3} / 6^{\prime}-\mathrm{OCH}_{3}\right), 3.83\left(\mathrm{~s}, 3 \mathrm{H}, \quad 4^{\prime}-\mathrm{OCH}_{3}\right) ;{ }^{13} \mathrm{C}$ NMR $\left(100 \mathrm{MHz}, \mathrm{CHCl}_{3}-d\right) \delta 162.0(\mathrm{Im}-\mathrm{C}-2), 159.5\left(\mathrm{C}-4^{\prime}\right), 159.2$ (C-2'/C-6'), 143.9 (C-1), 128.1 (C-3/C-5), 126.7 (C-4), $126.2(\mathrm{C}-2 / \mathrm{C}-6), 102.4\left(\mathrm{C}-1^{\prime}\right), 90.2\left(\mathrm{C}-3^{\prime} / \mathrm{C}-5^{\prime}\right), 57.7$ (Im$\mathrm{C}-4 / \mathrm{Im}-\mathrm{C}-5), 55.9\left(2^{\prime}-\mathrm{OCH}_{3} / 6^{\prime}-\mathrm{OCH}_{3}\right), 55.5\left(4^{\prime}-\mathrm{OCH}_{3}\right)$; $v_{\max }$ (ATR) $31523024290116231588 \mathrm{~cm}^{-1}$; HRMS (m/ z): Calcd. for $\mathrm{C}_{24} \mathrm{H}_{24} \mathrm{~N}_{2} \mathrm{O}_{3}[\mathrm{M}+\mathrm{H}]^{+}: 388.1839$, Found. 388.1787 .

(4R,5R)-2-(4-nitrophenyl)-4,5-diphenyl-imidazoline (3) Pale yellow $(26 \%$ yield $) ; \quad \mathrm{Mp}=200-202{ }^{\circ} \mathrm{C} ; \quad{ }^{1} \mathrm{H} \quad \mathrm{NMR}$ $\left(400 \mathrm{MHz}, \mathrm{DMSO}-d_{6}\right) \delta 8.35\left(\mathrm{~d}, J=8.9 \mathrm{~Hz}, 2 \mathrm{H}, \mathrm{H}-3^{\prime} / \mathrm{H}-\right.$ $\left.5^{\prime}\right), 8.26\left(\mathrm{~d}, J=8.9 \mathrm{~Hz}, 2 \mathrm{H}, \mathrm{H}-2^{\prime} / \mathrm{H}-6^{\prime}\right), 7.36(\mathrm{~m}, 1 \mathrm{H}, \mathrm{H}-4)$, 7.33 (m, 2H, H-2/H-6), 7.26 (m, 2H, H-3/H-5), 4.96 (d, $J=8.4 \mathrm{~Hz}, 1 \mathrm{H}, \mathrm{Im}-\mathrm{H}-4), 4.74(\mathrm{~d}, J=8.4 \mathrm{~Hz}, 1 \mathrm{H}, \mathrm{Im}-\mathrm{H}-$ 5,) ;{ }^{13} \mathrm{C}$ NMR (100 MHz, DMSO-d $\left.d_{6}\right) \delta 160.8$ (Im-C-2), $148.7\left(\mathrm{C}-4^{\prime}\right), 143.6(\mathrm{C}-1), 136.0\left(\mathrm{C}-1^{\prime}\right), 128.7\left(\mathrm{C}-2^{\prime} / \mathrm{C}-6^{\prime}\right)$, 126.7 (C-4), 126.2 (C-2/C-6), 123.6 (C-3/C-5), 79.6 (Im-C4), 69.3 (Im-C-5); v $\max$ (ATR) $3124302729301592 \mathrm{~cm}^{-1}$; HRMS (m/z): Calcd. for $\mathrm{C}_{21} \mathrm{H}_{17} \mathrm{~N}_{3} \mathrm{O}_{2} \quad[\mathrm{M}+\mathrm{H}]^{+}$: 343.1385, Found. 343.1321.

(4R,5R)-2-(4-methylphenyl)-4,5-diphenyl-imidazoline (4) [22] Pale yellow (60\% yield); $\mathrm{Mp}=138-140{ }^{\circ} \mathrm{C} ;{ }^{1} \mathrm{H}$ NMR $\left(400 \mathrm{~Hz}\right.$, DMSO- $\left.d_{6}\right) \delta 7.91(\mathrm{~d}, J=8.2 \mathrm{~Hz}, 2 \mathrm{H}$, H-2'/H-6'), 7.37 (m, 2H, H-3/H-5), 7.29 (m, 2H, H-3'/H$\left.5^{\prime}\right), 7.28$ (m, 2H, H-2/H-6), 7.27 (m, 1H, H-4), 4.76 (br s, $2 \mathrm{H}, \mathrm{Im}-\mathrm{H}-4 / \mathrm{Im}-\mathrm{H}-5), 2.39$ (s, $\left.1 \mathrm{H}, 4-\mathrm{CH}_{3}\right) ;{ }^{13} \mathrm{C} \mathrm{NMR}$ $\left(100 \mathrm{MHz}, \mathrm{DMSO}-d_{6}\right) \delta 162.2$ (Im-C-2), $144.4(\mathrm{C}-1)$, $140.4\left(\mathrm{C}-4^{\prime}\right), 129.0\left(\mathrm{C}-1^{\prime}\right), 128.6\left(\mathrm{C}-3^{\prime} / \mathrm{C}-5^{\prime}\right), 128.3(\mathrm{C}-3 / \mathrm{C}-$ 5), $127.3\left(\mathrm{C}-2^{\prime} / \mathrm{C}-6^{\prime}\right), 126.9$ (C-4), 126.5 (C-2/C-6), 70.9 (Im-C-4/Im-C-5), $21.0\left(4^{\prime}-\mathrm{CH}_{-3}\right) ; v_{\max }$ (ATR) 31423025 $291716241597 \mathrm{~cm}^{-1}$; HRMS (m/z): Calcd. for $\mathrm{C}_{22} \mathrm{H}_{20} \mathrm{~N}_{2}$ $[\mathrm{M}+\mathrm{H}]^{+}$: 312.1706, Found. 312.1626.

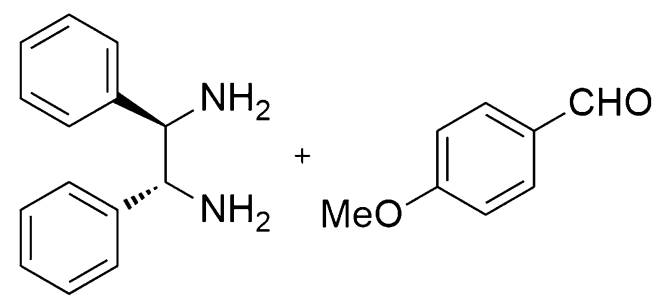

Scheme 1 The synthetic procedure for diphenyl imidazolines
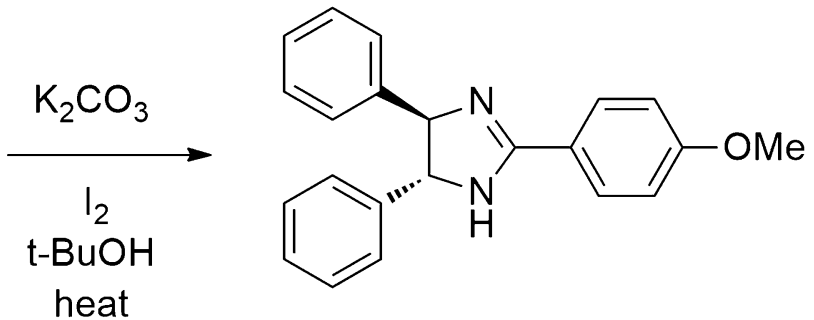
(4R,5R)-2-(4-bromophenyl)-4,5-diphenyl-imidazoline (5) White $\left(25 \%\right.$ yield); $\mathrm{Mp}=179-182{ }^{\circ} \mathrm{C} ;{ }^{1} \mathrm{H}$ NMR $\left(400 \mathrm{MHz}, \mathrm{DMSO}-d_{6}\right) \delta 8.02\left(\mathrm{~d}, J=8.1 \mathrm{~Hz}, 1 \mathrm{H}, \mathrm{H}-2^{\prime}\right)$, $7.51\left(\mathrm{~m}, 1 \mathrm{H}, \mathrm{H}-3^{\prime}\right), 7.36\left(\mathrm{~m}, 2 \mathrm{H}, \mathrm{H}-3^{\prime} / \mathrm{H}-5^{\prime}\right), 7.32(\mathrm{~m}, 1 \mathrm{H}$, H-4), 7.28 (m, 2H, H-2/H-6), 4.78 (s, 2H, Im-H-4/Im-H-5); ${ }^{13} \mathrm{C}$ NMR (100 MHz, DMSO- $\left.d_{6}\right) \delta 162.3$ (Im-C-2), 144.3 (C-1), $130.6\left(\mathrm{C}-1^{\prime}\right), 130.2\left(\mathrm{C}-4^{\prime}\right), 128.5(\mathrm{C}-3 / \mathrm{C}-5), 128.3$

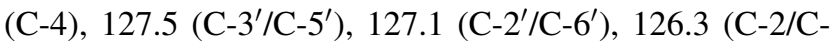
6), 76.8 (Im-C-4), 70.7 (Im-C-5); $v_{\max }$ (ATR) 31593027 $29061610 \mathrm{~cm}^{-1}$; HRMS (m/z): Calcd. for $\mathrm{C}_{21} \mathrm{H}_{17} \mathrm{BrN}_{2}$ $[\mathrm{M}+\mathrm{H}]^{+}:$376.0664, Found. 376.0575.

(4R,5R)-4,5-diphenyl-2-(thiophen-3-yl)-4,5-dihydro-1H-imidazole (6) Ivory (79\% yield); $\mathrm{Mp}=174-176{ }^{\circ} \mathrm{C} ;{ }^{1} \mathrm{H}$ NMR (400 MHz, DMSO- $\left.d_{6}\right) \delta 8.14(\mathrm{dd}, J=1.3,3.0 \mathrm{~Hz}$, $\left.1 \mathrm{H}, \mathrm{H}-2^{\prime}\right), 7.63\left(\mathrm{dd}, J=3.0,4.9 \mathrm{~Hz}, 1 \mathrm{H}, \mathrm{H}-4^{\prime}\right), 7.60$ (dd, $\left.J=1.3,4.9 \mathrm{~Hz}, 1 \mathrm{H}, \mathrm{H}-5^{\prime}\right), 7.36$ (m, 2H, H-3/H-5), 7.28 (m, 1H, H-4), 7.27 (m, 2H, H-2/H-6), 4.74 (s, 2H, Im-H-4/ Im-H-5); ${ }^{13} \mathrm{C}$ NMR (100 MHz, DMSO- $\left.d_{6}\right) \delta 158.5$ (Im-C2), 144.1 (C-1), 132.8 (C-1' $), 128.3$ (C-3/C-5), 127.1 (C-4), $127.1\left({\mathrm{C}-5^{\prime}}^{\prime}\right), 126.9\left(\mathrm{C}-2^{\prime}\right), 126.5\left(\mathrm{C}-4^{\prime}\right), 126.2$ (C-2/C-6), 79.1 (Im-C-4/Im-C-5); $v_{\max }$ (ATR) 315930242893 $1607 \mathrm{~cm}^{-1}$, HRMS (m/z): Calcd. for $\mathrm{C}_{19} \mathrm{H}_{16} \mathrm{~N}_{2} \mathrm{~S}$ $[\mathrm{M}+\mathrm{H}]^{+}$: 304.1107, Found. 304.1034.

(4R,5R)-2-(4-chlorophenyl)-4,5-diphenyl-imidazoline (7) Ivory ( $81 \%$ yield); $\mathrm{Mp}=168-172{ }^{\circ} \mathrm{C}$; ${ }^{1} \mathrm{H} \mathrm{NMR}$ (400 MHz, DMSO- $d_{6}$ ) $\delta 8.03$ (d, $J=8.5 \mathrm{~Hz}, 2 \mathrm{H}, \mathrm{H}-2^{\prime} / \mathrm{H}-$ 6), 7.56 (d, $\left.J=8.5 \mathrm{~Hz}, 2 \mathrm{H}, \mathrm{H}-3^{\prime} / \mathrm{H}-5^{\prime}\right), 7.37$ (m, 2H, H-3/ $\mathrm{H}-5), 7.28$ (m, 2H, H-2/H-6), 7.28 (m, 1H, H-4), 4.78 (s, $2 \mathrm{H}, \mathrm{Im}-\mathrm{H}-4 / \mathrm{Im}-\mathrm{H}-5) ;{ }^{13} \mathrm{C}$ NMR $\left(100 \mathrm{MHz}, \mathrm{DMSO}-d_{6}\right) \delta$ 161.3 (Im-C-2), 144.0 (C-1), 135.3 (C-4'), 129.2 (C-2'/C$\left.6^{\prime}\right), 129.0\left(\mathrm{C}-1^{\prime}\right), 128.5$ (C-3/C-5), $128.4\left(\mathrm{C}-3^{\prime} / \mathrm{C}-5^{\prime}\right), 127.1$ (C-4), 126.4 (C-2/C-6), 74.3 (Im-C-4/Im-C-5); $v_{\max }$ (ATR) $3152302429171622 \mathrm{~cm}^{-1}$; HRMS (m/z): Calcd. for $\mathrm{C}_{21} \mathrm{H}_{17} \mathrm{ClN}_{2}[\mathrm{M}+\mathrm{H}]^{+}:$332.1157, Found. 332.1080.

(4R,5R)-2-(4-fluorophenyl)-4,5-diphenyl-imidazoline (8) Ivory (41\% yield); $\mathrm{Mp}=186-189{ }^{\circ} \mathrm{C} ;{ }^{1} \mathrm{H}$ NMR $(400 \mathrm{MHz}$, DMSO- $\left.d_{6}\right) \delta 8.07\left(\mathrm{~d}, J=8.8 \mathrm{~Hz}, 2 \mathrm{H}, \mathrm{H}-2^{\prime} / \mathrm{H}-6\right), 7.37$ (m, 2H, H-3/H-5), 7.33 (d, $\left.J=8.8 \mathrm{~Hz}, 2 \mathrm{H}, \mathrm{H}-3^{\prime} / \mathrm{H}-5^{\prime}\right), 7.32$ (m, 2H, H-2/H-6), 7.25 (m, 1H, H-4), 4.88 (d, $J=7.2 \mathrm{~Hz}$, 1H, Im-H-4), 4.67 (d, $J=7.2 \mathrm{~Hz}, 1 \mathrm{H}, \mathrm{Im}-\mathrm{H}-5) ;{ }^{13} \mathrm{C} \mathrm{NMR}$ (100 MHz, DMSO- $\left.d_{6}\right) \delta 164.7$ (C-4'), 161.3 (Im-C-2), 144.0 (C-1), 129.8 (C-2'/C-6'), 128.4 (C-3/C-5), 126.7 (C4), $126.7\left(\mathrm{C}-1^{\prime}\right), 126.1$ (C-2/C-6), $115.2\left(\mathrm{C}-3^{\prime} / \mathrm{C}-5^{\prime}\right), 79.5$ (Im-C-4), 69.3 (Im-C-5); $v_{\max }$ (ATR) 312430272920 $1618 \mathrm{~cm}^{-1}$; HRMS (m/z): Calcd. for $\mathrm{C}_{21} \mathrm{H}_{17} \mathrm{FN}_{2}$ $[\mathrm{M}+\mathrm{H}]^{+}:$316.1463, Found. 316.1376.

(4R,5R)-2-(pyridine-2-yl)-4,5-diphenyl-imidazoline [23] Yellow (62\% yield); $\mathrm{Mp}=122-125{ }^{\circ} \mathrm{C} ;{ }^{1} \mathrm{H}$ NMR $\left(400 \mathrm{MHz}, \mathrm{DMSO}-d_{6}\right) \delta 8.69\left(\mathrm{~d}, J=4.8 \mathrm{~Hz}, 1 \mathrm{H}, \mathrm{H}-3^{\prime}\right)$, $8.18\left(\mathrm{~d}, J=7.9 \mathrm{~Hz}, 1 \mathrm{H}, \mathrm{H}-6^{\prime}\right), 7.95(\mathrm{td}, J=1.7,7.9 \mathrm{~Hz}$, $\left.1 \mathrm{H}, \mathrm{H}-5^{\prime}\right), 7.56\left(\mathrm{dd}, J=4.7,7.8 \mathrm{~Hz}, 1 \mathrm{H}, \mathrm{H}-4^{\prime}\right), 7.36(\mathrm{~m}$,
2H, H-3/H-5), 7.28 (m, 1H, H-4), 7.27 (m, 2H, H-2/H-6), 4.81 (br s, 2H, Im-H-4/Im-H-5); ${ }^{13} \mathrm{C}$ NMR $(100 \mathrm{MHz}$, DMSO- $\left.d_{6}\right) \delta 162.2(\mathrm{Im}-\mathrm{C}-2), 148.9\left(\mathrm{C}-3^{\prime}\right), 148.5\left(\mathrm{C}-1^{\prime}\right)$, 144.0 (C-1), $137.0\left({\mathrm{C}-5^{\prime}}^{\prime}\right), 128.5$ (C-3/C-5), 127.1 (C-4), 126.4 (C-2/C-6), $125.5\left({\mathrm{C}-4^{\prime}}^{\prime}\right), 122.5\left(\mathrm{C}-6^{\prime}\right), 69.0$ (Im-C-4/ Im-C-5); $v_{\max }$ (ATR) $3281302429111602 \mathrm{~cm}^{-1}$; HRMS (m/z): Calcd. for $\mathrm{C}_{20} \mathrm{H}_{17} \mathrm{~N}_{3}[\mathrm{M}+\mathrm{H}]^{+}:$299.1518, Found. 299.1422 .

(4R,5R)-2-(pyridine-3-yl)-4,5-diphenyl-imidazoline (10) Yellow $(23 \%$ yield $) ; \mathrm{Mp}=133-136{ }^{\circ} \mathrm{C} ;{ }^{1} \mathrm{H}$ NMR $(400 \mathrm{MHz}$, Acetone- $\left.d_{6}\right) \delta 9.26\left(\mathrm{dd}, J=0.7,2.3 \mathrm{~Hz}, 1 \mathrm{H}, \mathrm{H}-2^{\prime}\right), 8.71$ $\left(\mathrm{dd}, J=1.7,4.8 \mathrm{~Hz}, 1 \mathrm{H}, \mathrm{H}-4^{\prime}\right), 8.39(\mathrm{dt}, J=1.9,8.0 \mathrm{~Hz}$, $\left.1 \mathrm{H}, \mathrm{H}-6^{\prime}\right), 7.48$ (ddd, $\left.J=0.8,4.8,7.9 \mathrm{~Hz}, 1 \mathrm{H}, \mathrm{H}-5^{\prime}\right), 7.37$ (m, 2H, H-3/H-5), 7.34 (m, 1H, H-4), 7.31 (m, 2H, H-2/H6), 4.89 (s, 2H, Im-H-4/Im-H-5); ${ }^{13} \mathrm{C}$ NMR (100 MHz, Acetone- $\left.d_{6}\right) \delta 160.4(\mathrm{Im}-\mathrm{C}-2), 151.0\left(\mathrm{C}-4^{\prime}\right), 148.3\left(\mathrm{C}-2^{\prime}\right)$,

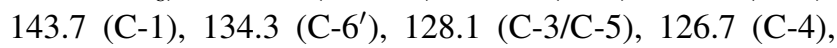

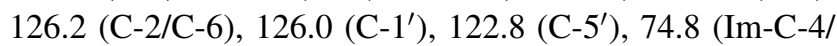
Im-C-5); $v_{\max }\left(\right.$ ATR) $3171303029031599 \mathrm{~cm}^{-1}$; HRMS $(\mathrm{m} / \mathrm{z})$ : Calcd. for $\mathrm{C}_{20} \mathrm{H}_{17} \mathrm{~N}_{3}[\mathrm{M}+\mathrm{H}]^{+}:$299.1524, Found. 299.1422 .

(4R,5R)-2,4,5-tris(4-methoxyphenyl)-4,5-dihydro-1H-imidazole (11) Cloudy yellow oil (27\% yield); $\mathrm{Mp}=150$ $154{ }^{\circ} \mathrm{C} ;{ }^{1} \mathrm{H}$ NMR (400 MHz, DMSO-d 6 ) $\delta 7.92 \quad(\mathrm{~d}$, $\left.J=8.8 \mathrm{~Hz}, 2 \mathrm{H}, \mathrm{H}-2^{\prime} / \mathrm{H}-6^{\prime}\right), 7.16$ (d, $J=8.4 \mathrm{~Hz}, 2 \mathrm{H}, \mathrm{H}-2 /$ H-6), 7.02 (d, $\left.J=8.8 \mathrm{~Hz}, 2 \mathrm{H}, \mathrm{H}-3^{\prime} / \mathrm{H}-5^{\prime}\right), 6.91$ (d, $J=8.4 \mathrm{~Hz}, 2 \mathrm{H}, \mathrm{H}-3 / \mathrm{H}-5), 4.63$ (br s, 2H, Im-H-4/Im-H-5), $3.82\left(\mathrm{~s}, 3 \mathrm{H}, 4^{\prime}-\mathrm{OCH}_{3}\right), 3.74\left(\mathrm{~s}, 3 \mathrm{H}, 4-\mathrm{OCH}_{3}\right) ;{ }^{13} \mathrm{C} \mathrm{NMR}$ $\left(100 \mathrm{MHz}, \mathrm{DMSO}-d_{6}\right) \delta 161.5$ (Im-C-2), $161.0\left(\mathrm{C}-4^{\prime}\right)$, 158.6 (C-4), 136.3 (C-1), $129.0\left(\mathrm{C}-2^{\prime} / \mathrm{C}-6^{\prime}\right), 127.5$ (C-2/C6), $122.8\left(\mathrm{C}-1^{\prime}\right), 113.8$ (C-3/C-5), 113.6 (C-3'/C-5'), 79.1 $(\mathrm{Im}-\mathrm{C}-4 / \mathrm{Im}-\mathrm{C}-5), \quad 55.3 \quad\left(4^{\prime}-\mathrm{OCH}_{3}\right), \quad 55.0 \quad\left(4-\mathrm{OCH}_{3}\right)$; $v_{\max }$ (ATR) $31613005292828381610 \mathrm{~cm}^{-1}$; HRMS $(\mathrm{m} / \mathrm{z})$ : Calcd. for $\mathrm{C}_{24} \mathrm{H}_{24} \mathrm{~N}_{2} \mathrm{O}_{3}[\mathrm{M}+\mathrm{H}]^{+}:$388.1846, Found. 388.1787.

(4R,5R)-2-(naphthalene-1-yl)-4,5-diphenyl-imidazoline (12) Ivory (79\% yield); $\mathrm{Mp}=196-200{ }^{\circ} \mathrm{C}$; ${ }^{1} \mathrm{H} \mathrm{NMR}$ $\left(400 \mathrm{MHz}, \mathrm{DMSO}-d_{6}\right) \delta 9.05(\mathrm{dd}, J=1.6,7.6 \mathrm{~Hz}, 1 \mathrm{H}$, H-8 $8^{\prime}, 8.08$ (d, $\left.J=8.2 \mathrm{~Hz}, 1 \mathrm{H}, \mathrm{H}-4^{\prime}\right), 8.01(\mathrm{~d}, J=7.3 \mathrm{~Hz}$, $\left.1 \mathrm{H}, \mathrm{H}-5^{\prime}\right), 7.99\left(\mathrm{dd}, J=1.2,7.1 \mathrm{~Hz}, 1 \mathrm{H}, \mathrm{H}-2^{\prime}\right), 7.62(\mathrm{~m}$, 1H, H-3') 7.60 (m, 1H, H-7'), 7.59 (m, 1H, H-6'), 7.40 (m, 2H, H-3/H-5), 7.38 (m, 2H, H-2/H-6), 7.32 (m, 1H, H-4), 4.87 (s, 2H, Im-H-4/Im-H-5); ${ }^{13} \mathrm{C}$ NMR $(100 \mathrm{MHz}$, DMSO- $\left.d_{6}\right) \delta 163.2(\mathrm{Im}-\mathrm{C}-2), 144.2(\mathrm{C}-1), 133.4\left(\mathrm{C}-1^{\prime}\right)$, $130.7\left(\mathrm{C}-9^{\prime}\right), 130.4\left(\mathrm{C}-4^{\prime}\right), 128.6(\mathrm{C}-3 / \mathrm{C}-5), 128.3\left(\mathrm{C}-5^{\prime}\right)$, $128.2\left(\mathrm{C}-10^{\prime}\right), 127.7\left(\mathrm{C}-7^{\prime}\right), 127.2\left(\mathrm{C}-2^{\prime}\right), 127.2(\mathrm{C}-4), 126.5$ (C-2/C-6), $126.3\left(\mathrm{C}-8^{\prime}\right), 126.1\left(\mathrm{C}-6^{\prime}\right), 125.0\left(\mathrm{C}-3^{\prime}\right), 73.7$ (Im-C-4/Im-C-5); $v_{\max }$ (ATR) $3136302729201563 \mathrm{~cm}^{-1}$; HRMS (m/z): Calcd. for $\mathrm{C}_{25} \mathrm{H}_{20} \mathrm{~N}_{2}[\mathrm{M}+\mathrm{H}]^{+}: 348.1715$, Found. 348.1626. 
Table 1 The names and structures of 14 diphenyl imidazolines and their half-maximal cell growth inhibitory concentrations $\left(\mathrm{GI}_{50}\right)$

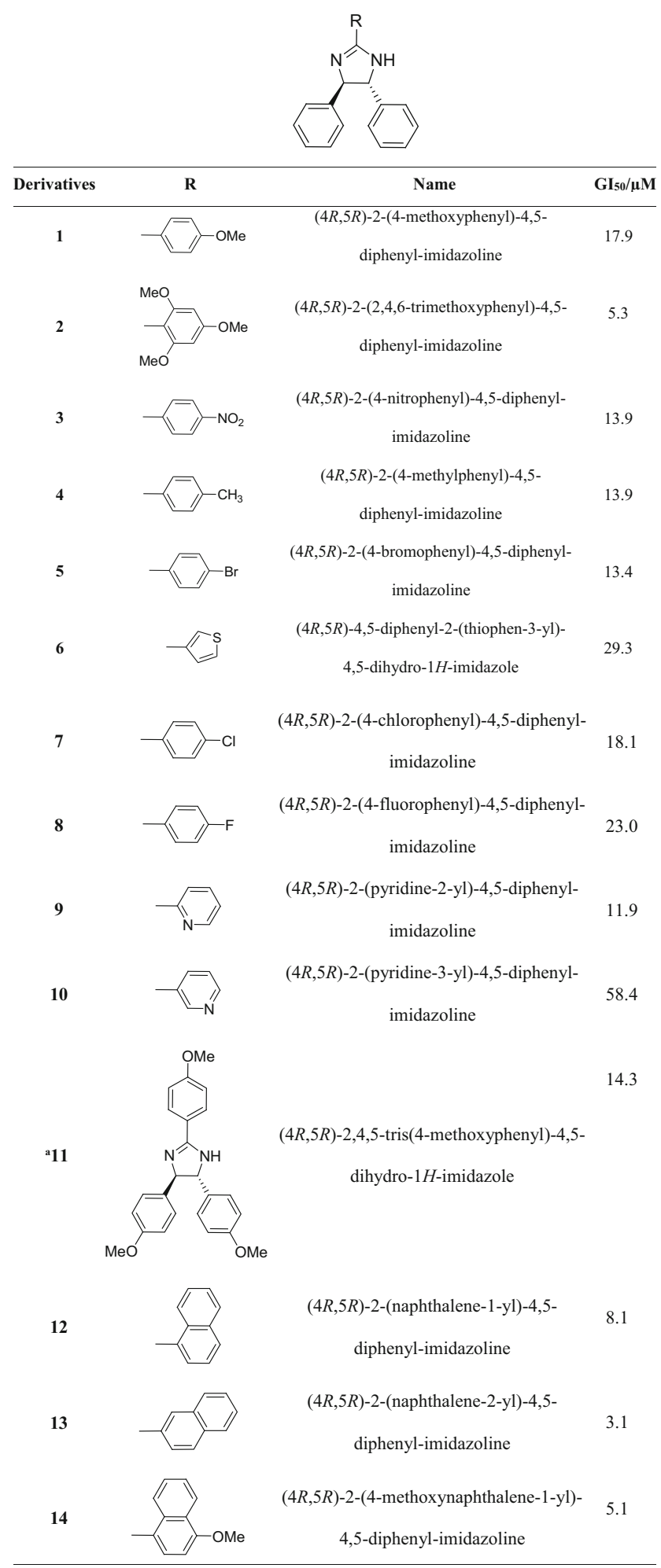

${ }^{a}$ Does not show R, but does a complete structure
(4R,5R)-2-(naphthalene-2-yl)-4,5-diphenyl-imidazoline (13) Pearl white (9\% yield); $\mathrm{Mp}=70-76{ }^{\circ} \mathrm{C} ;{ }^{1} \mathrm{H}$ NMR $\left(400 \mathrm{MHz}, \mathrm{DMSO}-d_{6}\right) \delta 8.57\left(\mathrm{~s}, 1 \mathrm{H}, \mathrm{H}-1^{\prime}\right), 8.16$ (dd, $\left.J=1.6,8.7 \mathrm{~Hz}, 1 \mathrm{H}, \mathrm{H}-3^{\prime}\right), 8.01\left(\mathrm{~d}, J=7.8 \mathrm{~Hz}, 1 \mathrm{H}, \mathrm{H}-5^{\prime}\right)$, $\left.7.99\left(\mathrm{~m}, 1 \mathrm{H}, \mathrm{H}-3^{\prime}\right), 7.61\left(\mathrm{~m}, 1 \mathrm{H}, \mathrm{H}-6^{\prime}\right), 7.59 \mathrm{~m}, 1 \mathrm{H}, \mathrm{H}-7^{\prime}\right)$, 7.37 (m, 2H, H-3/H-5), 7.34 (m, 2H, H-2/H-6), 7.31 (m, 1H, H-4), 4.84 (s, 2H, Im-H-4/Im-H-5); ${ }^{13} \mathrm{C} \quad \mathrm{NMR}$ $\left(100 \mathrm{MHz}, \mathrm{DMSO}-d_{6}\right) \delta 162.4$ (Im-C-2), 144.2 (C-1), $133.8\left(\mathrm{C}-10^{\prime}\right), 132.4\left({\mathrm{C}-9^{\prime}}^{\prime}\right), 128.5(\mathrm{C}-3 / \mathrm{C}-5), 128.4\left(\mathrm{C}-8^{\prime}\right)$, $127.8\left(\mathrm{C}-2^{\prime}\right), 127.6\left(\mathrm{C}-4^{\prime}\right), 127.3(\mathrm{C}-4), 127.3\left(\mathrm{C}-7^{\prime}\right), 127.1$ $\left(\mathrm{C}-1^{\prime}\right), 126.7\left({\mathrm{C}-5^{\prime}}^{\prime}\right), 126.7\left(\mathrm{C}-6^{\prime}\right), 126.5(\mathrm{C}-2 / \mathrm{C}-6), 124.8$ (C-3'), 73.3 (Im-C-4/Im-C-5); $v_{\max }$ (ATR) 314230272895 $1605 \mathrm{~cm}^{-1}$; HRMS (m/z): Calcd. for $\mathrm{C}_{25} \mathrm{H}_{20} \mathrm{~N}_{2}[\mathrm{M}+\mathrm{H}]^{+}$: 348.1716, Found. 348.1626.

(4R,5R)-2-(4-methoxynaphthalene-1-yl)-4,5-diphenyl-imidazoline (14) White (11\% yield); $\mathrm{Mp}=172-176{ }^{\circ} \mathrm{C} ;{ }^{1} \mathrm{H}$ NMR (400 MHz, DMSO- $\left.d_{6}\right) \delta 9.23(\mathrm{dm}, J=8.2 \mathrm{~Hz}, 1 \mathrm{H}$, $\left.\mathrm{H}-8^{\prime}\right), \quad 8.26\left(\mathrm{dm}, \quad J=8.1 \mathrm{~Hz}, 1 \mathrm{H}, \mathrm{H}-5^{\prime}\right), 7.98 \quad(\mathrm{~d}$, $\left.J=8.1 \mathrm{~Hz}, 1 \mathrm{H}, \mathrm{H}-2^{\prime}\right), 7.61(\mathrm{ddd}, J=1.2,7.1,8.2 \mathrm{~Hz}, 1 \mathrm{H}$, $\left.\mathrm{H}-7^{\prime}\right), 7.56\left(\mathrm{ddd}, J=1.1,7.1,8.1 \mathrm{~Hz}, 1 \mathrm{H}, \mathrm{H}-6^{\prime}\right), 7.40(\mathrm{~m}$, 2H, H-3/H-5), 7.36 (m, 2H, H-2/H-6), 7.36 (m, 1H, H-4), 7.07 (d, $\left.J=8.1 \mathrm{~Hz}, 1 \mathrm{H}, \mathrm{H}-3^{\prime}\right), 4.99(\mathrm{~d}, J=6.3 \mathrm{~Hz}, 1 \mathrm{H}$, Im-H-4), 4.66 (d, $J=6.3 \mathrm{~Hz}, 1 \mathrm{H}, \mathrm{Im}-\mathrm{H}-5), 4.04$ (s, 3H, 4' $\left.\mathrm{OCH}_{3}\right) ;{ }^{13} \mathrm{C}$ NMR $\left.(100 \mathrm{MHz} \text {, DMSO-d })_{6}\right) \delta 163.2(\mathrm{Im}-\mathrm{C}-$ 2), $156.4\left(\mathrm{C}-4^{\prime}\right), 144.3(\mathrm{C}-1), 131.9\left(\mathrm{C}-9^{\prime}\right), 128.6\left(\mathrm{C}-2^{\prime}\right)$, 128.4 (C-3/C-5), 127.1 (C-4), 126.7 (C-8'), 126.5 (C-2/C6), $126.3\left(\mathrm{C}-7^{\prime}\right), 125.5\left(\mathrm{C}-6^{\prime}\right), 125.0\left(\mathrm{C}-10^{\prime}\right), 121.6\left(\mathrm{C}-5^{\prime}\right)$, $120.1\left(\mathrm{C}-1^{\prime}\right), 103.5\left(\mathrm{C}-3^{\prime}\right), 80.6$ (Im-C-4), 68.5 (Im-C-5), $55.9\left(4^{\prime}-\mathrm{OCH}_{3}\right) ; v_{\max }(\mathrm{ATR}) 3161302929381582 \mathrm{~cm}^{-1}$; HRMS (m/z): Calcd. for $\mathrm{C}_{26} \mathrm{H}_{22} \mathrm{~N}_{2} \mathrm{O}[\mathrm{M}+\mathrm{H}]^{+}: 378.1837$, Found. 378.1732.

\section{Identification of diphenyl imidazoline derivatives}

The structures and names of the diphenyl imidazolines synthesized in this work are listed in Table 1. The compounds were dissolved in deuterated dimethyl sulfoxide (DMSO-d $\left.{ }_{6}\right)$, chloroform $\left(\mathrm{CHCl}_{3}-\mathrm{d}\right)$, and acetone $\left(\mathrm{CH}_{3-}\right.$ $\mathrm{COCH}_{3}-\mathrm{d}_{6}$ ) for nuclear magnetic resonance (NMR) spectroscopy and adjusted to a concentration of approximately $50 \mathrm{mM}$. These solutions were transferred to 2.5-mm NMR tubes. All NMR spectra were collected using an Avance 400 spectrometer system (9.4 T; Bruker, Karlsruhe, Germany) at $25{ }^{\circ} \mathrm{C}$. The NMR experimental conditions used are similar to those employed in a previous study [24]. To confirm the structures of the diphenyl-imidazoline derivatives, ultraperformance liquid chromatography-hybrid quadrupole-time-of-flight mass spectrometry was performed using a Waters Acquity UPLC system (Waters Corp., Milford, MA) with the help of Prof. Choong Hwan Lee at Konkuk University, Korea [25]. All high-resolution mass spectrometric (HRMS) data consisted of $\mathrm{M}+\mathrm{H}$ ions. 
Melting points and IR spectra were measured using a MelTemp II (LabX, Midland, ON, Canada) and an FT-IR 4200 spectrometer (JASCO, Easton, MD) equipped with an ATR (Attenuated Total Reflection, ATR PR0450-S), respectively [26]. The melting points and spectroscopic data for the 14 diphenyl imidazoline derivatives obtained from NMR spectroscopy, HRMS, and IR are summarized in the references and notes.

\section{Biological evaluation of diphenyl imidazoline derivatives}

HCT 116 human colorectal cancer cells were used for the cytotoxicity tests of the diphenyl imidazoline derivatives. The long-term clonogenic survival data were collected in the absence or presence of different concentrations $(0,1,5$, 10 , and $20 \mu \mathrm{M})$ of the 14 derivatives. The procedures adhered to previously reported methods [27]. The results were analyzed using densitometry (MultiGauge, Fujifilm, Japan), and the $\mathrm{GI}_{50}$ values were determined using SigmaPlot software (SYSTAT, Chicago, IL, USA) (Table 1) [28].

For the detection of MDM2 activity, the phosphorylation of the MDM2 protein at S166 was measured by immunoblotting analysis. HCT 116 cells were treated with $40 \mu \mathrm{M}$ derivative 13, (4R,5R)-2-(naphthalene-2-yl)-4,5diphenyl-imidazoline, for various time periods $(0,3,6,12$,

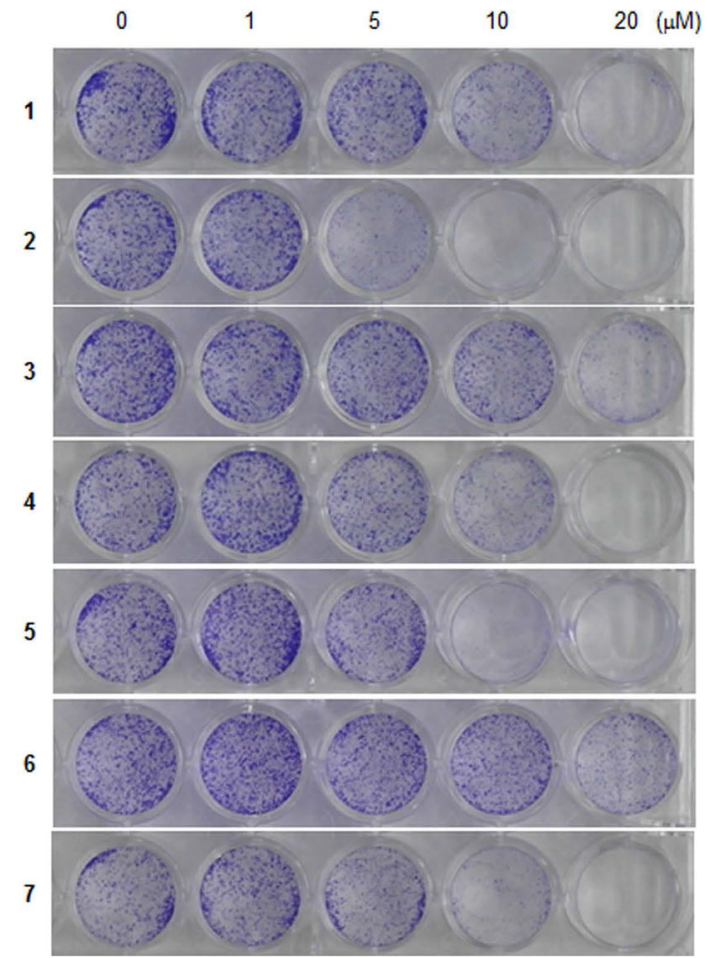

or $24 \mathrm{~h})$ and then extracted with cell lysis buffer $(25 \mathrm{mM}$ Tris- $\mathrm{HCl} \mathrm{pH} 7.5,1 \%$ Triton $\mathrm{X}-100,10 \%$ glycerol, $150 \mathrm{mM} \mathrm{NaCl}, 1 \mathrm{mM} \mathrm{Na} \mathrm{VO}_{4}$, and $10 \mathrm{mg} / \mathrm{mL}$ leupeptin). Protein extracts were electrophoresed on a $10 \%$ SDSpolyacrylamide gel, transferred to nitrocellulose membranes, and incubated with anti-phospho-MDM2 antibody (Cell Signaling Technology, Beverly, MA, USA). The glyceraldehyde phosphate dehydrogenase (GAPDH) antibody from Santa Cruz Biotechnology (Santa Cruz, CA, USA) was used as an internal control. The blots were developed using the enhanced chemiluminescence detection system (GE Healthcare, Piscataway, NJ, USA) [29].

\section{Statistical analysis}

Statistical analyses were conducted using a one-way analysis of variance (ANOVA), followed by Kruskal-Wallis test, by using GraphPad Instat software (La Jolla, CA). All experiments were performed in triplicate [30].

\section{In silico docking}

For in silico docking experiments, a three-dimensional (3D) structure of derivative $\mathbf{1 3}$ was obtained from the crystal structure of a ligand for 1ttv.pdb and modified [31]. From the 3D structures of MDM2 deposited in the PDB, 4ode.pdb was selected for this experiment because it has

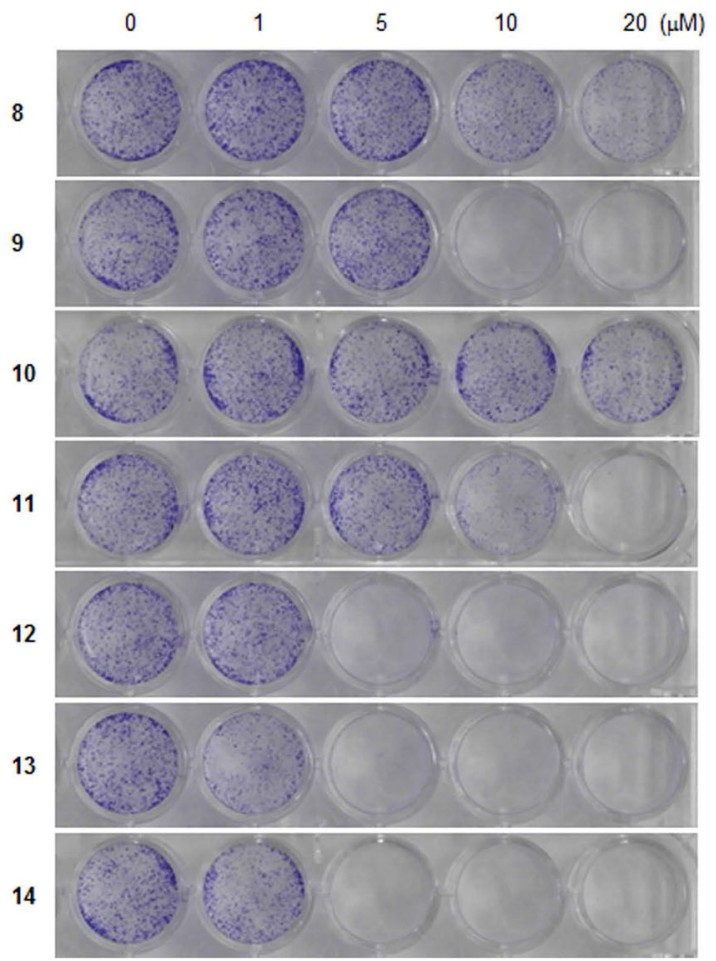

Fig. 1 The clonogenic long-term survival assays of cells treated with 14 diphenyl imidazolines at different concentrations $(0,1,5,10$, and $20 \mu \mathrm{M})$ 
Fig. 2 The half-maximal cell growth inhibitory concentration $\left(\mathrm{GI}_{50}\right)$ values in the HCT 116 human colorectal cancer cell lines of 14 diphenyl imidazolines with error bars

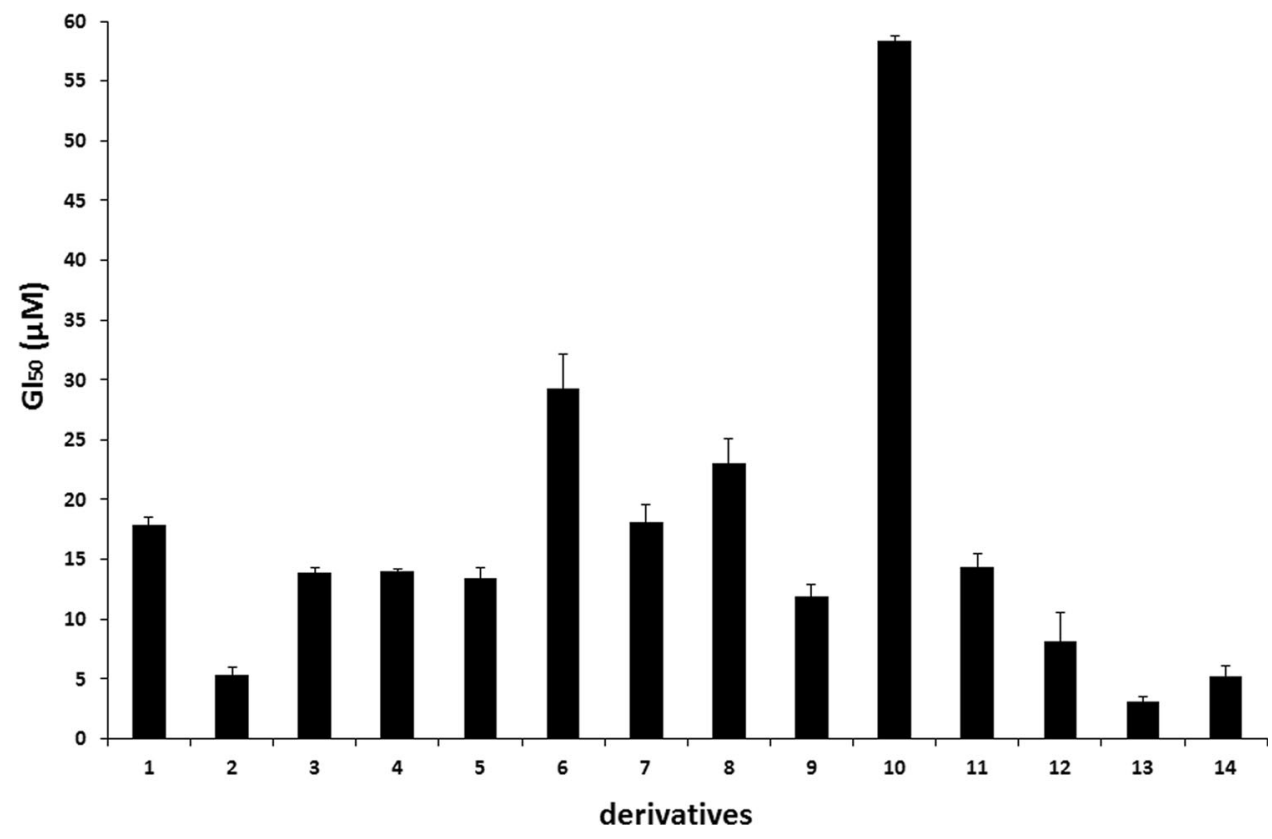

the longest chain of the N-terminal p53 interaction domain (6-110 residues) at a high resolution (1.8 ̊) [32]. In silico docking experiments were performed on an Intel Core 2 Quad Q6600 (2.4 GHz) Linux PC with Sybyl 7.3 software (Tripos) [33], and LigPlot software was used to identify the binding sites [34]. All 3D images were generated using the PyMOL software (The PyMOL Molecular Graphics System, Version 1.0r1, Schrödinger, LLC).

\section{Results and discussion}

The cytotoxicities of the diphenyl imidazolines were measured using a clonogenic long-term survival assay, as shown in Fig. 1. The $\mathrm{GI}_{50}$ values were in the range

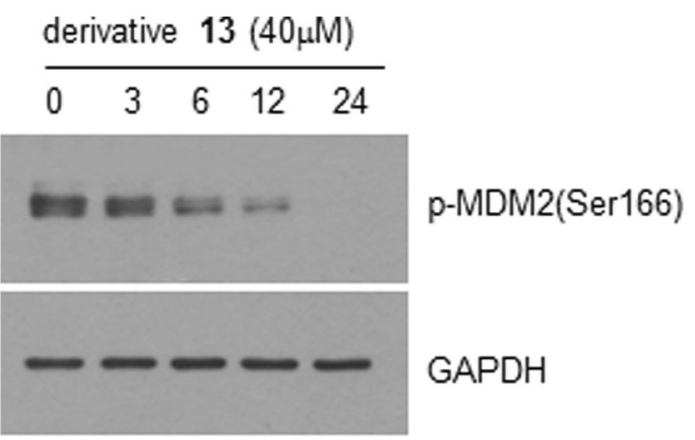

Fig. 3 Effect of derivative $\mathbf{1 3}$ on the phosphorylation of MDM2 at S166. HCT 116 cells were treated with $40 \mu \mathrm{M}$ derivative $\mathbf{1 3}$ for different time periods. Whole cell lysates were separated by $10 \%$ SDS-PAGE, and the phosphorylation status of MDM2 on S166 was determined by immunoblotting. GAPDH was used as an internal control
3.1-58.4 $\mu \mathrm{M}$, as listed in Table 1, and the bar graph (including errors) is shown in Fig. 2.

Derivatives 12 and 13 both contained a 2-naphthalenyl group, but the former was 2-naphthalen-1-yl and the latter was 2-naphthalen-2-yl. The $\mathrm{GI}_{50}$ values of $8.1 \mu \mathrm{M}$ and $3.1 \mu \mathrm{M}$, respectively, indicated that the 2-naphthalen-1-yl group was a better pharmacophore than the 2-naphthalen1-yl group was. Derivatives $\mathbf{5}, \mathbf{7}$, and $\mathbf{8}$ contained the halogens bromine, chlorine, and fluorine at the para position of the 2-phenyl group, respectively, with $\mathrm{GI}_{50}$ values of $13.4 \mu \mathrm{M}, 18.1 \mu \mathrm{M}$, and $23.0 \mu \mathrm{M}$, respectively. Consequently, it was shown that the electronegativity affected the cytotoxicity. Derivative 1, with a $p$-methoxy phenyl group, showed a higher $\mathrm{GI}_{50}$ value than did derivative $\mathbf{4}$ that contained $p$-toluene. A comparison of the derivatives $\mathbf{1 ,} \mathbf{2}$,

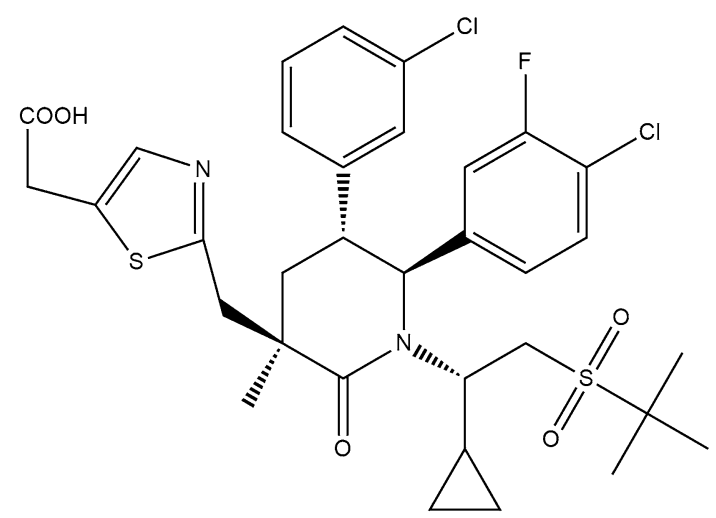

Fig. 4 The ligand contained in the MDM2 protein (4ode.pdb), (2\{[(3R,5R,6S)-1-[(1S)-2-(tert-butylsulfonyl)-1-cyclopropylethyl]-6-(4chloro-3-fluorophenyl)-5-(3-chlorophenyl)-3-methyl-2-oxopiperidin3-yl]methyl \}-1,3-thiazol-5-yl) acetic acid 


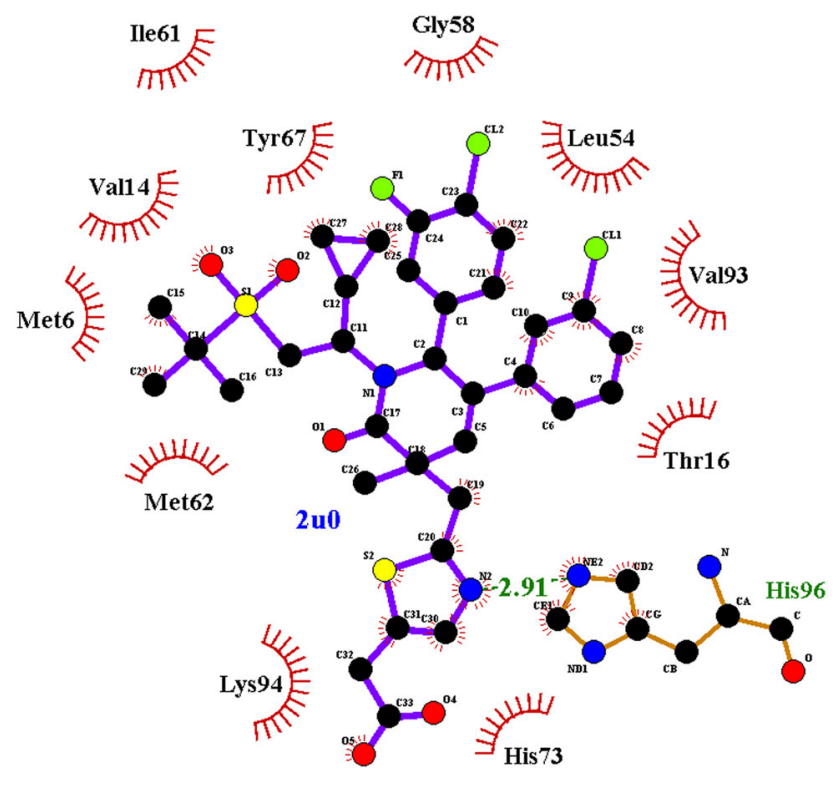

4ode

Fig. 5 The residues participating in the binding sites of the original ligand-MDM2 complex were analyzed using the LigPlot program

and 11 revealed that the cytotoxicity was improved by an increase in the number of the methoxy groups of diphenyl imidazolines. This phenomenon agreed with the behavior of derivatives 12 and 14. Derivative 14, with a 4-methoxynaphthalenyl group, showed a lower $\mathrm{GI}_{50}$ than did derivative 12 without the 4-methoxy group. The $\mathrm{GI}_{50}$ value

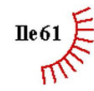<smiles>C=CC=CC</smiles>

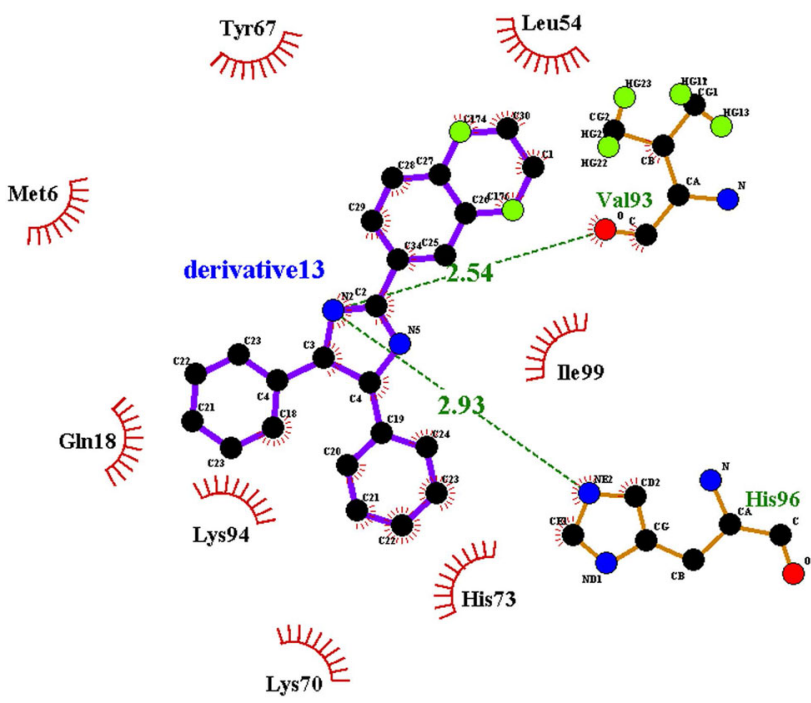

Fig. 6 The residues participating in the binding sites of the derivative 13-MDM2 complex were analyzed using the LigPlot program. The residues in half circles form hydrophobic interactions with the ligand of derivative 9, 2-((4R,5R)-4,5-diphenyl-imidazolin-2yl)pyridine, was better than that of derivative $\mathbf{1 0}$, 3-((4R,5R)-4,5-diphenyl-imidazolin-2-yl)pyridine, which indicated that the 2-pyridinyl group was a better pharmacophore than the 3-pyridinyl group was. This phenomenon might be caused by the different distances between the nitrogen of pyridine and the nitrogens of imidazoline. Derivative 6, with a thiopen-3-yl group, and derivative 10, with a pyridin-3-yl group, showed less cytotoxicity than did derivative 9, with a pyridin-2-yl group. The distance between the two nitrogens of the former compound was $4.5 \AA$ and that of the latter was $3.0 \AA$.

The p53 protein functions as a tumor-suppressor gene, which plays a critical role in the induction of cell cycle arrest, DNA repair, and apoptosis after diverse DNA damage responses [35-37]. MDM2 is an oncoprotein that prevents apoptosis by inducing the degradation of p53 [38]. MDM2 is activated by the phosphorylation of S166, which is mediated by the MEK/ERK or PI3 K/AKT pathways $[39,40]$. As mentioned above, the anticancer effects of diphenyl imidazolines were attributable to their inhibitory effects against MDM2 [17-20]. To investigate whether the phosphorylation of MDM2 at S166 was affected by diphenyl imidazoline derivative $\mathbf{1 3}$ showing the most potent cell growth inhibitory effect, (4R,5R)-2-(naphthalene-2-yl)-4,5-diphenyl-imidazoline, HCT 116 cells were subjected to treatment with $40 \mu \mathrm{M}$ derivative $\mathbf{1 3}$ for different periods of time. The results indicated that the phosphorylation of MDM2 at S166 was decreased in a time-dependent manner (Fig. 3). Thus, derivative 13 efficiently inhibited the phosphorylation of MDM2 on S166.

In order to understand the binding mode of derivative 13, which showed the strongest cytotoxicity among the derivatives tested, an in silico docking experiment was carried out. Based on the LigPlot analysis of 4ode.pdb, the ligand (short name: 2U0), (2-\{[(3R,5R,6S)-1-[(1S)-2-(tert-butylsulfonyl)1-cyclopropylethyl]-6-(4-chloro-3-fluorophenyl)-5-(3-chlorophenyl)-3-methyl-2-oxopiperidin-3-yl]methyl \}-1,3-thiazol-5-yl) acetic acid (Fig. 4), was secured by 11 hydrophobic interactions (M6, V14, T16, L54, G58, I61, M62, Y67, H73, V93, and K94) and one H-bond (H-96) (Fig. 5).

The 12 residues in the binding pocket were used for the docking experiment of derivative 13. After docking the derivative 13 into MDM2, 30 complexes were generated from 30 docking iteration processes. The binding energy of the 30 complexes was in the range -17.80 to - $10.69 \mathrm{kcal} / \mathrm{mol}$. Among the 30 complexes, the seventh complex, with $-12.14 \mathrm{kcal} / \mathrm{mol}$ of binding energy, was selected to clarify the binding mode based on both the binding pose and the binding energy. This complex was analyzed using the LigPlot program. Twelve residues were involved in the binding of derivative 13 with MDM2: M6, 
Fig. 7 The slice view of binding pocket of MDM2 complex with (A) 2U0 and (B) derivative $\mathbf{1 3}$
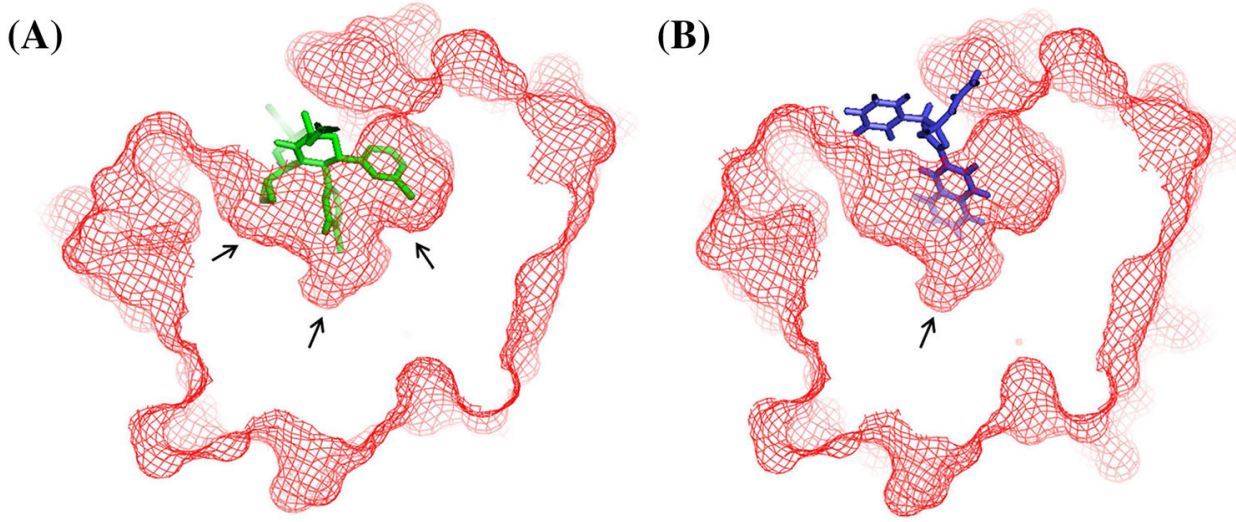

(A)

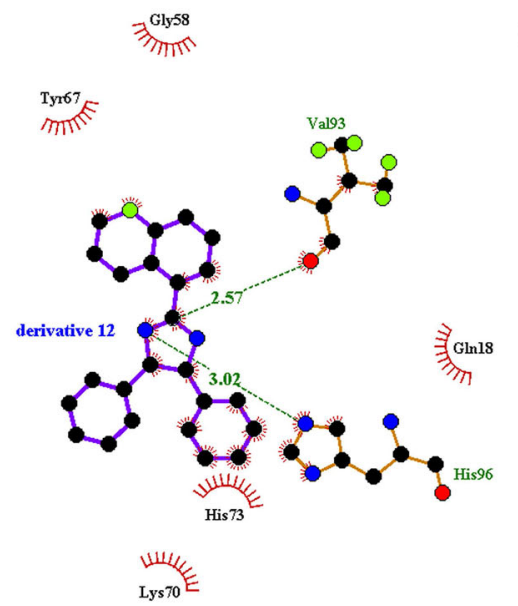

(B)

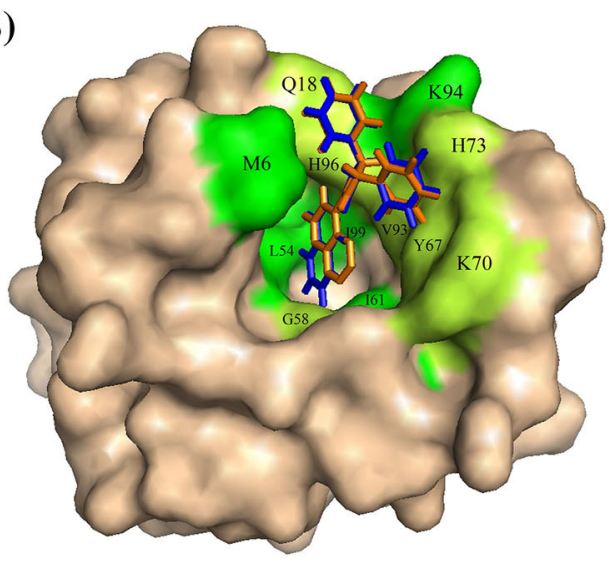

Fig. 8 (A) The residues participating in the binding sites of the derivative 12-MDM2 complex were analyzed using the LigPlot program, and (B) shows the binding modes of derivatives $\mathbf{1 2}$ (orange) and $\mathbf{1 3}$ (blue) with MDM2. The residues colored light green interacted with derivative $\mathbf{1 2}$ and all 12 residues interacted with derivative $\mathbf{1 3}$ derivative 12 (orange), which contains 2-naphthalen-1-yl. A hydrophobic interaction was observed between MDM2 and the 2-naphthalen-1-yl group of derivative $\mathbf{1 2}$.

As previously mentioned, derivative 2 , with tri-methoxy groups at the 2-phenyl ring, showed better inhibition compared with the activities of derivatives 1-11. This suggested that the bulky group at C-2 of imidazoline was necessary for docking into the binding pocket of MDM2.

In conclusion, we synthesized 14 diphenyl imidazoline derivatives and examined their cytotoxicities. Although the diphenyl imidazoline derivatives used in this study have only small structural differences, the $\mathrm{GI}_{50}$ values of the 14 derivatives in HCT 116 cells varied from 3.1 to $58.4 \mu \mathrm{M}$. Based on the results of SARs and in silico docking studies, we therefore proposed that bulky groups, such as naphthalene or a highly methoxylated phenyl ring, were favored at the C-2 position of imidazoline. Additionally, branched structures were suitable for tight binding because of the presence of three small pockets.

Acknowledgments This work was supported by the Priority Research Centers Program [NRF, 2009-0093824]. SY Shin was supported by the KU Research Professor Program of Konkuk University. 


\section{References}

1. Ueno M, Imaizumi K, Sugita T, Takata I, Takeshita M (1995) Effect of a novel anti-rheumatic drug, TA-383, on type II collagen-induced arthritis. Int J Immunopharmacol 17(7):597-603

2. Sugita T, Ueno M, Furukawa O, Murakami T, Takata I, Tosa T (1993) Effect of a novel anti-rheumatic drug, TA-383, on type II collagen-induced arthritis-suppressive effect of TA-383 on interleukin 6 production. Int J Immunopharmacol 15(4):515-519

3. Li YF, Gong ZH, Cao JB, Wang HL, Luo ZP, Li J (2003) Antidepressant-like effect of agmatine and its possible mechanism. Eur J Pharmacol 469(1-3):81-88

4. Rodriguez F, Rozas I, Kaiser M, Brun R, Nguyen B, Wilson WD, Garcia RN, Dardonville C (2008) New bis(2-aminoimidazoline) and bisguanidine DNA minor groove binders with potent in vivo antitrypanosomal and antiplasmodial activity. J Med Chem 51(4):909-923

5. Browne N, Hackenberg F, Streciwilk W, Tacke M, Kavanagh K (2014) Assessment of in vivo antimicrobial activity of the carbene silver(I) acetate derivative SBC3 using Galleria mellonella larvae. Biometals 27(4):745-752

6. Ueno M, Sugita T, Murakami T, Takata I (1997) The novel antirheumatic drug TA-383 has a macrophage migration enhancing activity. Jpn J Pharmacol 74(2):221-224

7. Shukla S, Bhalla M, Misra U, Mukerjee D, Saxsena AK, Sinha JN, Shanker K (1998) Cardiovascular effects of novel imidazoline congeners. Boll Chim Farm 137(7):229-232

8. Dardonville C, Goya P, Rozas I, Alsasua A, Martin MI, Borrego MJ (2000) New aromatic iminoimidazolidine derivatives as alpha1-adrenoceptor antagonists: a novel synthetic approach and pharmacological activity. Bioorg Med Chem 8(7):1567-1577

9. Marson CM, Matthews CJ, Atkinson SJ, Lamadema N, Thomas NS (2015) Potent and selective inhibitors of histone deacetylase-3 containing chiral oxazoline capping groups and a N-(2-aminophenyl)-benzamide binding unit. J Med Chem 58(17):6803-6818

10. Koga K, Honda K, Ando S, Harasawa I, Kamiya HO, Takano Y (2004) Intrathecal clonidine inhibits mechanical allodynia via activation of the spinal muscarinic M1 receptor in streptozotocininduced diabetic mice. Eur J Pharmacol 505(1-3):75-82

11. Cheng YF, Hu YZ, He QJ (2005) Synthesis and antitumor activity of arylsubstituted imidazolin-2-one derivatives. Yao Xue Xue Bao 40(8):711-716

12. American Cancer Society. www.cancer.org

13. Mosmann T (1983) Rapid colorimetric assay for cellular growth and survival: application to proliferation and cytotoxicity assays. J Immunol Methods 65(1-2):55-63

14. Cory AH, Owen TC, Barltrop JA, Cory JG (1991) Use of an aqueous soluble tetrazolium/formazan assay for cell growth assays in culture. Cancer Commun 3(7):207-212

15. Berridge MV, Tan AS (1993) Characterization of the cellular reduction of 3-(4,5-dimethylthiazol-2-yl)-2,5-diphenyltetrazolium bromide (MTT): subcellular localization, substrate dependence, and involvement of mitochondrial electron transport in MTT reduction. Arch Biochem Biophys 303(2):474-482

16. Franken NA, Rodermond HM, Stap J, Haveman J, van Bree C (2006) Clonogenic assay of cells in vitro. Nat Protoc 1(5):2315-2319

17. Zhang Z, Chu XJ, Liu JJ, Ding Q, Zhang J, Bartkovitz D, Jiang N, Karnachi P, So SS, Tovar C, Filipovic ZM, Higgins B, Glenn K, Packman K, Vassilev L, Graves B (2014) Discovery of potent and orally active p53-MDM2 inhibitors RO5353 and RO2468 for potential clinical development. ACS Med Chem Lett 5(2):124-127
18. Carry JC, Garcia-Echeverria C (2013) Inhibitors of the p53/hdm2 protein-protein interaction-path to the clinic. Bioorg Med Chem Lett 23(9):2480-2485

19. Ghosh P, Zhang J, Shi ZZ, Li K (2013) Synthesis and evaluation of an imidazole derivative-fluorescein conjugate. Bioorg Med Chem 21(8):2418-2425

20. Zhang Z, Ding Q, Liu JJ, Zhang J, Jiang N, Chu XJ, Bartkovitz D, Luk KC, Janson C, Tovar C, Filipovic ZM, Higgins B, Glenn K, Packman K, Vassilev LT, Graves B (2014) Discovery of potent and selective spiroindolinone MDM2 inhibitor, RO8994, for cancer therapy. Bioorg Med Chem 22(15):4001-4009

21. Dauwe C, Buddrus J (1995) Synthesis of enantiopure C-2-chiral amidines. Synthesis-Stuttgart 2:171-172

22. Ishihara M, Togo H (2006) An efficient preparation of 2-imidazolines and imidazoles from aldehydes with molecular iodine and (diacetoxyiodo)benzene. Synlett 2:227-230

23. Fujioka H, Murai K, Ohba Y, Hiramatsu A, Kita Y (2005) A mild and efficient one-pot synthesis of 2-dihydroimidazoles from aldehydes. Tetrahedron Lett 46(13):2197-2199

24. Yong Y, Ahn S, Hwang D, Yoon H, Jo G, Kim YH, Kim SH, Koh D, Lim Y (2013) 1H and 13C NMR spectral assignments of $2^{\prime}$ hydroxychalcones. Magn Reson Chem 51(6):364-370

25. Ahn S, Shin SY, Jung Y, Jung H, Kim BS, Koh D, Lim Y (2016) (1) H and (13) C NMR spectral assignments of novel flavonoids bearing benzothiazepine. Magn Reson Chem 54(5):382-390

26. Shin SY, Jung H, Ahn S, Hwang D, Yoon H, Hyun J, Yong Y, Cho HJ, Koh D, Lee YH, Lim Y (2014) Polyphenols bearing cinnamaldehyde scaffold showing cell growth inhibitory effects on the cisplatin-resistant A2780/Cis ovarian cancer cells. Bioorg Med Chem 22(6):1809-1820

27. Yoon H, Kim TW, Shin SY, Park MJ, Yong Y, Kim DW, Islam T, Lee YH, Jung KY, Lim Y (2013) Design, synthesis and inhibitory activities of naringenin derivatives on human colon cancer cells. Bioorg Med Chem Lett 23(1):232-238

28. Jung Y, Shin SY, Yong Y, Jung H, Ahn S, Lee YH, Lim Y (2015) Plant-derived flavones as inhibitors of aurora B kinase and their quantitative structure-activity relationships. Chem Biol Drug Des 85(5):574-585

29. Shin SY, Ahn S, Yoon H, Jung H, Jung Y, Koh D, Lee YH, Lim Y (2016) Colorectal anticancer activities of polymethoxylated 3-naphthyl-5-phenylpyrazoline-carbothioamides. Bioorg Med Chem Lett 26(17):4301-4309

30. Jung H, Shin SY, Jung Y, Tran TA, Lee HO, Jung KY, Koh D, Cho SK, Lim Y (2015) Quantitative relationships between the cytotoxicity of flavonoids on the human breast cancer stem-like cells MCF7-SC and their structural properties. Chem Biol Drug Des 86(4):496-508

31. Fry DC, Emerson SD, Palme S, Vu BT, Liu CM, Podlaski F (2004) NMR structure of a complex between MDM2 and a small molecule inhibitor. J Biomol NMR 30(2):163-173

32. Gonzalez AZ, Li Z, Beck HP, Canon J, Chen A, Chow D, Duquette J, Eksterowicz J, Fox BM, Fu J, Huang X, Houze J, Jin L, Li Y, Ling Y, Lo MC, Long AM, McGee LR, McIntosh J, Oliner JD, Osgood T, Rew Y, Saiki AY, Shaffer P, Wortman S, Yakowec P, Yan X, Ye Q, Yu D, Zhao X, Zhou J, Olson SH, Sun D, Medina JC (2014) Novel inhibitors of the MDM2-p53 interaction featuring hydrogen bond acceptors as carboxylic acid isosteres. J Med Chem 57(7):2963-2988

33. Shin SY, Yoon H, Hwang D, Ahn S, Kim DW, Koh D, Lee YH, Lim Y (2013) Benzochalcones bearing pyrazoline moieties show anti-colorectal cancer activities and selective inhibitory effects on aurora kinases. Bioorg Med Chem 21(22):7018-7024

34. Wallace AC, Laskowski RA, Thornton JM (1995) LIGPLOT: a program to generate schematic diagrams of protein-ligand interactions. Protein Eng 8(2):127-134 
35. Fridman JS, Lowe SW (2003) Control of apoptosis by p53. Oncogene 22(56):9030-9040

36. Yin Y, Tainsky MA, Bischoff FZ, Strong LC, Wahl GM (1992) Wild-type p53 restores cell cycle control and inhibits gene amplification in cells with mutant p53 alleles. Cell 70(6):937-948

37. Zhan Q (2005) Gadd45a, a p53- and BRCA1-regulated stress protein, in cellular response to DNA damage. Mutat Res 569(1-2):133-143
38. Kubbutat MH, Jones SN, Vousden KH (1997) Regulation of p53 stability by Mdm2. Nature 387(6630):299-303

39. Malmlof M, Roudier E, Hogberg J, Stenius U (2007) MEK-ERKmediated phosphorylation of Mdm2 at Ser-166 in hepatocytes. $\mathrm{Mdm} 2$ is activated in response to inhibited Akt signaling. J Biol Chem 282(4):2288-2296

40. Zhou BP, Liao Y, Xia W, Zou Y, Spohn B, Hung MC (2001) HER-2/neu induces p53 ubiquitination via Akt-mediated MDM2 phosphorylation. Nat Cell Biol 3(11):973-982 